\title{
Coherent Integration Loss Due to Nonstationary Phase Noise in High-Resolution Millimeter-Wave Radars
}

\author{
Chagai Levy*(D), Monika Pinchas (D) and Yosef Pinhasi \\ Department of Electrical and Electronic Engineering, Ariel University, Ariel 40700, Israel; \\ monika.pinchas@gmail.com (M.P.); yosip@ariel.ac.il (Y.P.) \\ * Correspondence: chagailevy@gmail.com
}

Citation: Levy, C.; Pinchas, M.;

Pinhasi, Y. Coherent Integration Loss Due to Nonstationary Phase Noise in High-Resolution Millimeter-Wave Radars. Remote Sens. 2021, 13, 1755. https://doi.org/10.3390/rs13091755

Academic Editor: Vito Pascazio

Received: 27 February 2021

Accepted: 27 April 2021

Published: 30 April 2021

Publisher's Note: MDPI stays neutral with regard to jurisdictional claims in published maps and institutional affiliations.

Copyright: (c) 2021 by the authors. Licensee MDPI, Basel, Switzerland. This article is an open access article distributed under the terms and conditions of the Creative Commons Attribution (CC BY) license (https:// creativecommons.org/licenses/by/ $4.0 /)$.

\begin{abstract}
Phase noise refers to the instability of an oscillator, which is the cause of instantaneous phase and frequency deviations in the carrier wave. This unavoidable instability adversely affects the performance of range-velocity radar systems, including synthetic aperture radars (SARs) and ground-moving target indicator (GMTI) radars. Phase noise effects should be considered in highresolution radar designs, operating in millimeter wavelengths and terahertz frequencies, due to their role in radar capability during the reliable identification of target location and velocity. In general, phase noise is a random process consisting of nonstationary terms. It has been shown that in order to optimize the coherent detection of stealthy, fast-moving targets with a low radar cross-section (RCS), it is required to evaluate the integration gain and to determine the incoherent noise effects for resolving target location and velocity. Here, we present an analytical expression for the coherent integration loss when a nonstationary phase noise is considered. A Wigner distribution was employed to derive the time-frequency expression for the coherent loss when nonstationary conditions were considered. Up to now, no analytical expressions have been developed for coherent integration loss when dealing with real nonstationary phase noise mathematical models. The proposed expression will help radar systems estimate the nonstationary integration loss and adjust the decision threshold value in order to maximize the probability of detection. The effect of nonstationary phase noise is demonstrated for studying coherent integration loss of high-resolution radar operating in the W-band. The investigation indicates that major degradation in the time-frequency coherent integration due to short-term, nonstationary phase noise instabilities arises for targets moving at low velocities and increases with range. Opposed to the conventional model, which assumes stationarity, a significant difference of up to $25 \mathrm{~dB}$ is revealed in the integration loss for radars operating in the millimeter wave regime. Moreover, for supersonic moving targets, the loss peaks at intermediate distances and then reduces as the target moves away.
\end{abstract}

Keywords: phase noise; W-band radar; Wigner-Ville distribution; atomic clocks; coherent integration

\section{Introduction}

In recent years, the usage of millimeter- and terahertz (THz)-wave radars is continuously growing. These systems require a very high phase accuracy in order to increase the resolution in the detection of low-radar-cross-section (RCS) moving targets. The probability of radar detection is normally enhanced by coherent time integration being performed on the quadrature detector components prior to the decision [1]. Such coherent (or predetection) integration preserves the phase within the received signal, revealing an increased signal-to-noise ratio (SNR) to improve the detection reliability and measurement accuracies [2]. Such a procedure is implemented in radars, sonar, communications, etc. The phase noise is the random jitter in the oscillator time-base generating the carrier wave [3]. Since the radar measures the respective time delay of the reflected signal, any deviation in the clock timing translates to randomness in the range and velocity measurement. In coherent detection, the noise manifests itself as a phase modulation, introducing 
errors in the delay time of the signal scattered from the target. This modulation then affects the resolution of the detection, potentially impacting target detectability and mensuration.

In [4], an analytic discrete form for the coherent integration gain in the presence of stationary white Gaussian phase noise was derived for radar, sonar, and communication links. Based on a generalized analysis of coherent integration loss, Yu et al. [2] proposed an analytic upper bound of the coherent integration loss for the stationary phase noise with symmetric Gaussian, uniform, and Laplace distributions. Obviously, coherent integration loss [2,4] is a time-invariant expression; thus, the SNR loss is time-independent-assuming that stationarity, i.e., constant phase variance, yields time-independence in coherent integration loss expressions [2,4]. Coherent acquisition cannot be integrated for an arbitrary period if stationarity is assumed [5]. In addition, frequency sources are exposed to a variety of environmental conditions, causing variations in the oscillator output signal [6]. These variations are expressed in phase instabilities and variations in the oscillator output signal are considered to be a stochastic process, which is a time-variant nonstationary process $[7,8]$.

Here, we propose a new expression for the coherent integration loss caused by nonstationary oscillator phase noise. To date, time-frequency analysis has not yet been used in the characterization of the radar coherent integration loss caused by nonstationary oscillator phase noise. In our new expression, we used a realistic mathematical model for the nonstationary phase noise. Our proposed expression can estimate the coherent integration loss in radar, sonar, and communication systems by revealing the time-varying frequencies generally seen in clock error noise under nonstationary conditions and in other platforms, such as systems under vibration or shocks. Moreover, we proposed a new technique that will allow usage of loss information in order to adjust the decision threshold. Such a technique can help radar systems to evaluate real-time estimation of the nonstationary integration loss and adjust the decision threshold value according to the estimation result in order to maximize the radar probability of detection.

The simulation results indicate that our new proposed expression can estimate the varying coherent integration loss in a W-band high-resolution radar system while the conventional stationary model cannot reveal those variations.

A short review of the quasi-monostatic radar model and the phase noise in oscillators is provided in Section 2. The mathematical model of nonstationary phase noise in oscillators is presented in Section 3. The proposed approach for coherent integration loss using the Wigner spectrum is presented in Section 4 . In Section 5, the high-resolution radar simulation results are presented. Section 6 summarizes the paper.

\section{The Quasi-Monostatic Radar Model}

A simplified block diagram of a radar transceiver that employs coherent integration followed by a threshold decision is shown in Figure 1.

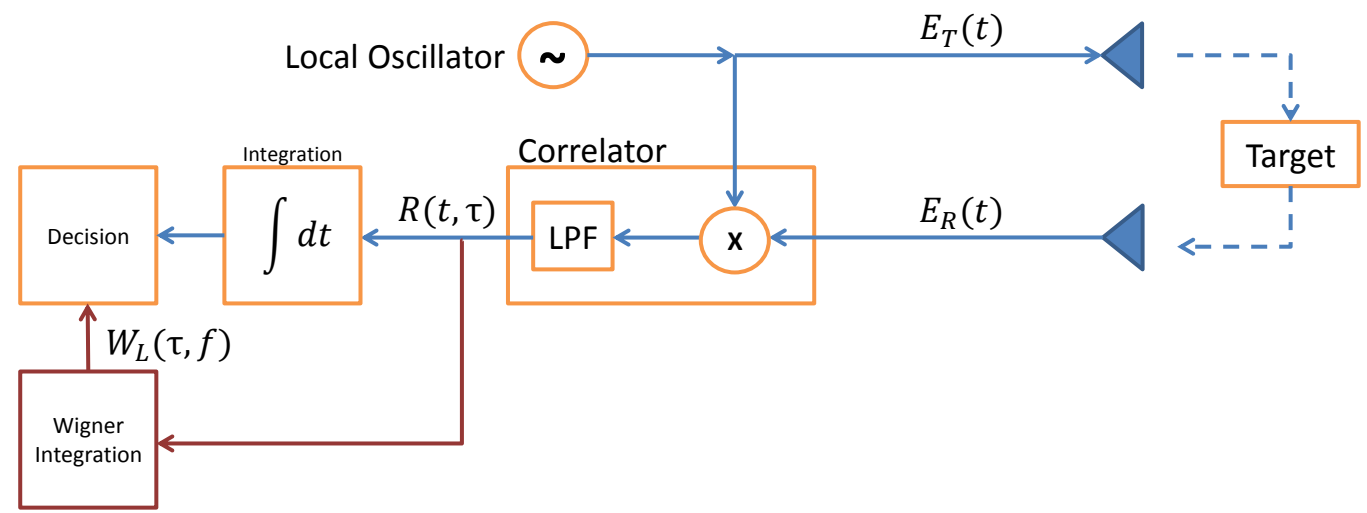

Figure 1. Simplified block diagram of a quasi-monostatic radar system with a threshold receiver and a coherent integration loss system. 
The transmitted signal $\widetilde{E}_{T}(t)$ generated by the radar's local oscillator, including the phase $\varphi(t)$ variations due to noise, is given by [6,9]

$$
\widetilde{E}_{T}(t)=A_{T} e^{j\left[2 \pi v_{0} t+\varphi(t)\right]},
$$

where $A_{T}$ is the amplitude and $v_{0}$ is the central frequency of the carrier wave. The received signal $\widetilde{E}_{R}(t)$ is merely a delayed version of the transmitted signal $\widetilde{E}_{T}(t)$, where $\tau$ is the echo delay time.

$$
\widetilde{E}_{R}(t)=A_{R} e^{j\left[2 \pi v_{0}(t-\tau)+\varphi(t-\tau)\right]},
$$

where $A_{R}$ is the amplitude of the reflected signal. The received signal $\widetilde{E}_{R}(t)$ is heterodyned with the transmitted one $\widetilde{E}_{T}(t)$, resulting in a complex stochastic correlation:

$$
R(t, \tau)=\widetilde{E}_{R}^{*}(t) \widetilde{E}_{T}(t)=A_{R} A_{T} e^{j\left[2 \pi v_{0} \tau+\varphi(t)-\varphi(t-\tau)\right]},
$$

with an expected value given by

$$
\bar{R}(t, \tau)=E\{R(t, \tau)\}=A_{R} A_{T} e^{j 2 \pi v_{0} \tau} E\left\{e^{j[\varphi(t)-\varphi(t-\tau)]}\right\} .
$$

The operation $E\{x\}$ denotes the expectation value of $x$. The effect of short-term, nonstationary phase deviation $\varphi(t)$ on the correlation (4) is analyzed in the following section. The "Correlator" block is responsible for extracting the intermediate frequency (IF) information. It is based on a heterodyne mixing of the received signal $\widetilde{E}_{R}(t)$ scattered from the target with the transmitted signal $\widetilde{E}_{T}(t)$ followed by a low-pass filter (LPF). The block "Integration" represents the coherent integration process that preserves the phase information of the IF signal by simply integrating the result over time. The unit "Decision" makes the decision about the existence of the target in space. Basically, it has some set threshold limit that is compared with the magnitude of the received signal. If the threshold value is surpassed by the output signal, then this shows that presence of the target. Otherwise, it is assumed that only the noise component is present in the space. Radar designers seek to maximize the probability of detection for a given probability of false alarm. Thus, the proposed "Wigner integration" block will help to estimate the integration loss caused by the nonstationary phase noise. Then, an adjustment to the threshold value (described above in the "Decision" block) can be made according to the estimation results.

\section{Nonstationary Phase Noise in Oscillators}

In this section, we review the mathematical model of nonstationary phase noise in oscillators in order to propose a novel model, which will take place in the following nonstationary coherent integration loss expression. The phase variation $\varphi(t)$ is given as a summation of seven terms [10,11]:

$\varphi(t)=2 \pi v_{0}\left[x_{0}+y_{0} t+a \frac{t^{2}}{2}+\mu_{3} \frac{t^{3}}{6}+\sigma_{1} B_{H_{1}}(t)+\sigma_{2} \int_{0}^{t} B_{H_{2}}(s) d s+\sigma_{3} \int_{0}^{t} \frac{(t-s)^{2}}{2} d B_{H_{3}}(s)\right]$,

where parameters are defined as follows:

1. $x_{0}$ and $y_{0}$ are the initial phase and frequency offsets, respectively.

2. $\quad a$ is defined as the frequency drift coefficient.

3. $\mu_{3}$ is a linear variation coefficient added to the frequency drift.

4. $\left\{B_{H_{i}}(t), t \geq 0\right\}, i=1,2,3$ are three independent, one-dimensional standard Wiener processes (Brownian motion) [10]. The Wiener process is often referred to as an integral of white noise, and is, in principle, a nonstationary process.

5. The diffusion coefficients $\sigma_{1}$ and $\sigma_{2}$ represent the white- and random walk-frequency noises, respectively [12].

6. $\sigma_{3}$ is related to the frequency drift. Since $\sigma_{3}$ is related to long-term frequency drift, it is not relevant to short-term analysis, and was thus neglected [10]. 
The phase variations $\varphi(t)$ can be divided into short- and long-term instabilities [10]. The long-term characteristics due to oscillator aging result in a very slow drift in the carrier frequency. This behavior is not relevant to radar scenarios, as it is only noticeable if the frequency is observed over hours and days, while the delay of the received signal is significantly shorter. Short-term phase noise is an essential issue when considering coherent integration. Therefore, hereafter, we concentrate on short-term instabilities only (6), since it is the influencing factor in coherent radar integration.

The stochastic short-term of the phase variations $\varphi(t)$ is given by

$$
\varphi(t)=2 \pi v_{0}\left[x_{0}+y_{0} t+\sigma_{1} B_{H_{1}}(t)+\sigma_{2} \int_{0}^{t} B_{H_{2}}(s) d s\right] .
$$

The resulted phase difference, which is required in Equation (4) for cross-correlation $\bar{R}(t, \tau)$, is then

$$
\varphi(t)-\varphi(t-\tau)=2 \pi v_{0}\left[y_{0} \tau+\sigma_{1} B_{H_{1}}(t)+\sigma_{2} \int_{0}^{t} B_{H_{2}}(s) d s-\sigma_{1} B_{H_{1}}(t-\tau)-\sigma_{2} \int_{0}^{t-\tau} B_{H_{2}}(s) d s\right] .
$$

\section{Coherent Integration Loss in the Presence Nonstationary Phase Noise}

A closed-form expression for the characterization of coherent integration loss due to nonstationary phase noise in local oscillators can now be derived. Our analytical derivation is based on the Wigner-Ville spectrum [13-15], which expends the spectral measures to contain the nonstationary characteristics of the oscillator phase variation. The Wigner-Ville distribution does not suffer from leakage effects as the short-time Fourier transformation (STFT) does. Hence, the Wigner-Ville distribution gives the best spectral resolution [13]. Therefore, in this paper, we used the Wigner-Ville distribution for time-frequency analysis. The integration loss is usually defined [4] as the ratio of the correlation $\bar{R}(t, \tau)$ in the presence of noise to the "pure" (noiseless) correlation $R_{0}(\tau)$ :

$$
\bar{L}(t, \tau)=\frac{\bar{R}(t, \tau)}{R_{0}(\tau)} .
$$

In the absence of phase noise (when $\varphi(t)=0$ ), the resulted correlation is

$$
R_{0}(\tau)=A_{R} A_{T} e^{j 2 \pi v_{0} \tau}
$$

Substituting (4) and (9) into the expression for the coherent integration loss (8) yields

$$
\bar{L}(t, \tau)=E\left\{e^{j[\varphi(t)-\varphi(t-\tau)]}\right\} .
$$

When nonstationary phase noise is present, the resulted $\bar{L}(t, \tau)$ is a time-varying stochastic process. The time-frequency transform of the nonstationary coherent integration loss, defined by (10), is the Wigner distribution [13-15], which is expressed via Fourier transformation:

$$
\bar{W}_{L}(\tau, f)=\int_{-\infty}^{\infty} \bar{L}(t, \tau) e^{-j 2 \pi f t} d t .
$$

While the power spectral density (PSD) is a result of time integration that assimilates the time domain, the Wigner distribution is a time-dependent measurement [15] that can reveal the phase variations of an oscillator in high-resolution radars.

In typical oscillators such as the temperature-compensated crystal oscillator (TCXO) and oven-controlled crystal oscillator (OCXO), for Rubidium and Cesium frequency standards, if $\sigma_{1}$ and $\sigma_{2}$, which represent the magnitude of the oscillator noise, are small enough with respect to 1 , then we can state that $\varphi(t)<<1, \varphi(t-\tau)<<1$ and make the following approximations: 


$$
\begin{aligned}
& \cos [\varphi(t)] \approx 1 \\
& \cos [-\varphi(t-\tau)] \approx 1 \\
& \sin [\varphi(t)] \approx \varphi(t) \\
& \sin [-\varphi(t-\tau)] \approx-\varphi(t-\tau) .
\end{aligned}
$$

Substituting (12) into (10) gives

$$
\bar{L}(t, \tau)=E\{1+j \varphi(t)-j \varphi(t-\tau)+\varphi(t) \varphi(t-\tau)\} .
$$

Then, substituting (13) into (11) gives

$$
\bar{W}_{L}(\tau, f)=\int_{-\infty}^{\infty} E\{1+j \varphi(t)-j \varphi(t-\tau)+\varphi(t) \varphi(t-\tau)\} e^{-j 2 \pi f t} d t .
$$

The phases $\varphi(t)$ and $\varphi(t-\tau)$ are standard Wiener processes with expected values $E\{\varphi(t)\}=E\{-\varphi(t-\tau)\}=0$. Consequently, the resulted $E\{j \varphi(t)-j \varphi(t-\tau)\}=0$, resulting in

$$
\bar{W}_{L}(\tau, f)=\int_{-\infty}^{\infty} E\{1+\varphi(t) \varphi(t-\tau)\} e^{-j 2 \pi f t} d t .
$$

In order to find $\bar{W}_{L}(\tau, f),(6)$ is substituted into (15) and the integral of the expectation value in (15) is solved (see Appendix A for a detailed derivation). Doing this yields a closed form time-frequency expression for the nonstationary coherent integration loss:

$$
\begin{aligned}
& \bar{W}_{L}(\tau, f)=\delta(f)+\left(2 \pi v_{0}\right)^{2}\left\{\frac{\sigma_{1}^{2}}{2}\left[|\tau| \delta(f)+\frac{1-e^{-j 2 \pi \tau f}}{2 \pi^{2} f^{2}}\right]+\frac{\sigma_{2}^{2}}{6} e^{-j 2 \pi f \tau}\left[\frac{-2 \pi^{2} f^{2} \tau^{2}+j 4 \pi f \tau+3}{4 \pi^{4} f^{4}}+\frac{\tau(\pi f \tau-j)}{2 \pi^{3} f^{3}}\right]\right. \\
& \left.+\frac{\sigma_{2}^{2}}{4}\left(\frac{3-j 2 \pi f \tau}{4 \pi^{4} f^{4}}\right)+\frac{\sigma_{2}^{2}}{12}\left[|\tau| \tau^{2} \delta(f)+\frac{e^{-j 2 \pi \tau f} \tau^{2}}{2 \pi^{2} f^{2}}-\frac{3}{4 \pi^{4} f^{4}}\right]\right\} .
\end{aligned}
$$

The resulted time-frequency coherent integration loss (16) is now applied to analyze high-resolution radars operating in the millimeter-wave regime.

\section{High-Resolution Terahertz Radar}

The radar presented in Figure 2 is a W-band ranging sensor module presented in [16]. This is a continuous-wave high-resolution radar operating at $94 \mathrm{GHz}$. The meaning of the phrase "high-resolution" refers to the high-resolution detection of the target's instantaneous velocity. The transmission at high carrier frequency compensates for employing a narrower temporal window in the Wigner spectrum, while maintaining the high velocity resolution [16]. In such extremely high frequencies, directive antennas with a relatively small aperture can be employed. The $94-\mathrm{GHz}$ carrier frequency is within the atmospheric $\mathrm{W}$-band transmission window, suffering relatively low atmospheric absorption. The lowmedium loss enables increasing the distance to the target, thereby enabling detection even during adverse weather conditions. The radar parameters are presented in detail in [16].

A schematic illustration of the radar is shown in Figure 3. A continuous carrier wave at $15.67 \mathrm{GHz}$ produced by the Agilent $83623 \mathrm{~B}$ frequency synthesizer is multiplied by a factor of 6 , resulting in a $94-\mathrm{GHz}$ carrier. A small amount of the generated power is coupled to the product detector of the receiving chain via a directional coupling port. Two identical horn lens antennas are employed for transmission and reception.

Figure 4 proposes a general geometric model for the transmitted and received signals' trajectories relative to the distance between the antennas $x=x_{1}+x_{2}$. 


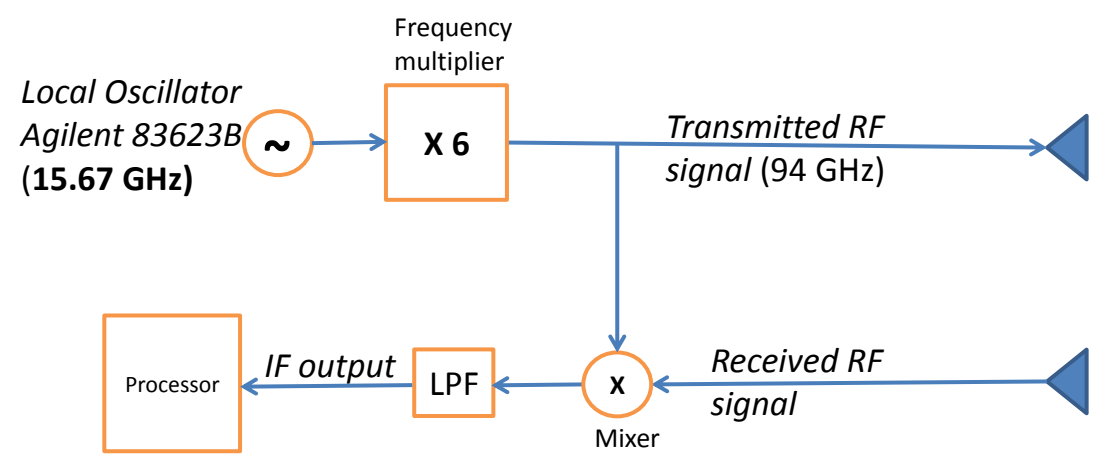

Figure 2. Block diagram of a W-band Doppler radar.

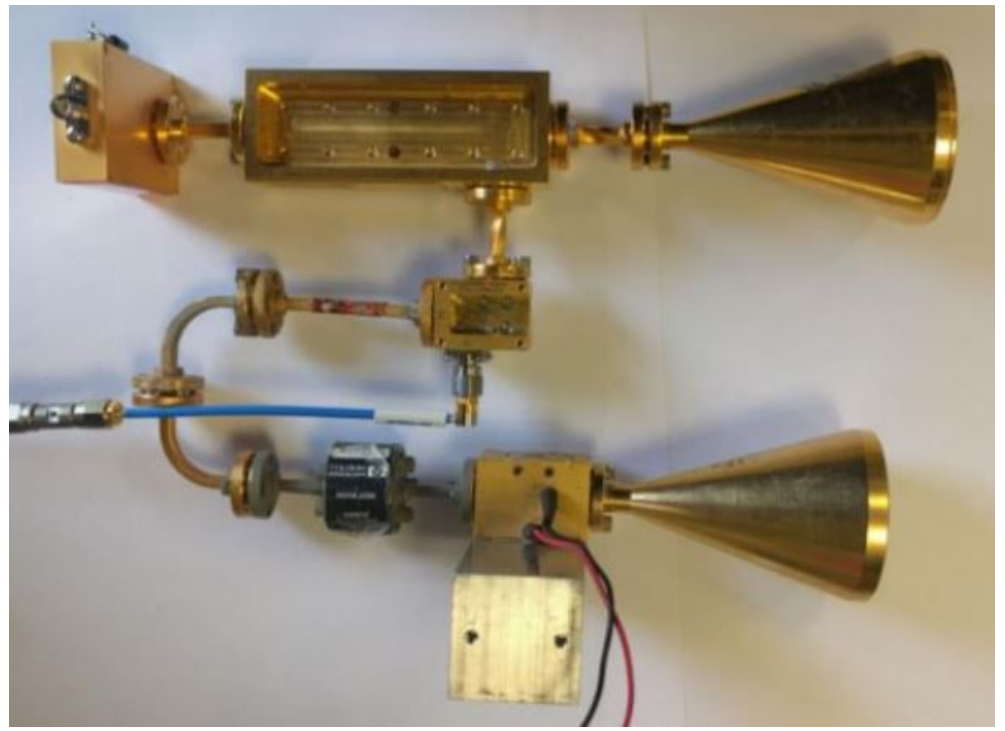

Figure 3. The continuous-wave micro-Doppler radar [16].

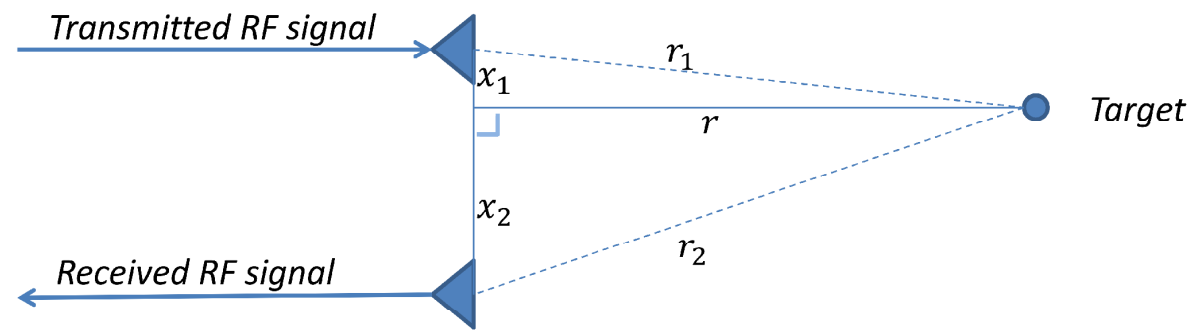

Figure 4. Simplified geometry model of a quasi-monostatic radar system.

The intermediate frequency (IF) product at the mixer's output is

$$
R(t, \tau)=\widetilde{E}_{R}^{*}(t) \widetilde{E}_{T}(t)=A_{R} A_{T} e^{j} \overbrace{\left[\frac{2 \pi v_{0}}{c} \cdot\left[r_{1}(t)+r_{2}(t)\right]+\varphi(t)-\varphi(t-\tau)\right]}^{\Psi(t)},
$$

where $c$ is the speed of light. $r_{1}(t)$ and $r_{2}(t)$ are the distances from the transmitting antenna to the moving target and from the moving target to the receiving antenna, respectively. According to the geometry model of a quasi-monostatic radar presented in Figure 4, we can present the angle $\Psi(t)$ relative to the distance between the antennas:

$$
\Psi(t)=\frac{2 \pi v_{0}}{c} \cdot\left[\sqrt{r^{2}(t)+x_{1}^{2}}+\sqrt{r^{2}(t)+x_{2}^{2}}\right]+\varphi(t)-\varphi(t-\tau) .
$$


$r_{1}(t)$ and $r_{2}(t)$ can be written in terms of their radial velocities $v_{r_{1}}(t)$ and $v_{r_{2}}(t)$, respectively, as

$$
\begin{aligned}
& r_{1}(t)=r_{1_{0}}+\int_{-\infty}^{t} v_{r_{1}}\left(t^{\prime}\right) d t^{\prime} \\
& r_{2}(t)=r_{2_{0}}+\int_{-\infty}^{t} v_{r_{2}}\left(t^{\prime}\right) d t^{\prime},
\end{aligned}
$$

where $r_{1_{0}}$ and $r_{2_{0}}$ are the initial distances from the transmitting antenna to the moving target and from the moving target to the receiving antenna, respectively. The IF product obtained at the mixer output is the derivative of the angle $\Psi(t)$ :

$$
f_{I F}(t, \tau)=\frac{1}{2 \pi} \frac{d \Psi(t)}{d t}=\frac{v_{0}}{c}\left[\dot{r}_{1}(t)+\dot{r_{2}}(t)\right]+\frac{1}{2 \pi}[\dot{\varphi}(t)-\dot{\varphi}(t-\tau)],
$$

where the "over-dot" $\dot{x}(t)$ denotes a time derivative of $x(t)$. Please note that Balal et al. [16] presented two experiments where the distance from the antennas to the target was greater by about two orders of magnitude from the distance between the antennas, thus, $r>>x_{1}$ and $r>>x_{2}$ and we can state that $r(t) \approx r_{1}(t) \approx r_{2}(t)$. Now, we can write (20) in terms of $\dot{r}(t)$ :

$$
f_{I F}(t, \tau)=2 \frac{v_{0}}{c} \dot{r}(t)+\frac{1}{2 \pi}[\dot{\varphi}(t)-\dot{\varphi}(t-\tau)]
$$

The time delay $\tau=2 r_{0} / c$ is expressed in terms of the distance $r_{0}$ to the target. Substituting the time delay and the target radial velocity $v_{r}(t)=\dot{r}(t)$ into (21) results in

$$
f_{I F}(t, \tau)=2 \frac{v_{0}}{c} \cdot v_{r}(t)+\frac{1}{2 \pi}\left[\dot{\varphi}(t)-\dot{\varphi}\left(t-\frac{2 r_{0}}{c}\right)\right] .
$$

In the last equation, the Doppler frequency shift can be detected due to the instantaneous radial velocity $v_{r}(t)$ of the target according to

$$
f_{d}(t)=\frac{2 v_{0}}{c} \cdot v_{r}(t)
$$

The resulting Doppler frequency shift $f_{d}(t)$ in the IF output is shown to be proportional to the target radial velocity. A target moving at the radial speed of $v_{r}=1000 \mathrm{~m} / \mathrm{s}$, for instance, will be detected by a $94-\mathrm{GHz} \mathrm{W}$-band radar as a Doppler shift tone equal to $f_{d}=626 \mathrm{KHz}$, whereas the same $\mathrm{W}$-band radar will detect a target moving ten times slower $\left(v_{r}=100 \mathrm{~m} / \mathrm{s}\right)$ as a Doppler shift tone equal to $f_{d}=62.6 \mathrm{KHz}$.

The preceding time-frequency analysis was then used to identify nonstationary instabilities in the phase of the frequency synthesizer to follow its spectral variations in the time domain. An estimation of the Agilent 83623B [17] diffusion coefficients $\sigma_{1}, \sigma_{2}$ can be made using the power law phase noise spectra function [12]. Under quiescent conditions, we found $\sigma_{1}=10^{-4}, \sigma_{2}=10^{-6}$.

Please note that the proposed method is also available for realistic state-of-the-art microwave signal sources with an integrated Voltage Controlled Oscillator (VCO), e.g., the Analog Devices ADF4371 [18]. The ADF4371 allows implementation of a fractional-N or Integer N phase-locked loop (PLL) frequency synthesizer with a wide-band output frequency range of $62.5 \mathrm{MHz}$ to $32 \mathrm{GHz}$. This range allows the usage in this integrated circuit (ADF4371) as the local oscillator of the W-band Doppler radar presented in Figure 2.

Figure $5 \mathrm{a}$ is the resulted three-dimensional plot of the nonstationary coherent integration loss $(16)$ in the time-frequency $(\tau, f)$ domain. Figure $5 \mathrm{~b}$ presents the corresponding contour plot after converting it to range $(r)$ and radial velocity $\left(v_{r}\right)$. At low Doppler frequencies (corresponding to small velocities), the local oscillator of the radar presents higher intensity of phase noise that increases the coherent integration loss. As the target velocity increases, the phase noise power decreases, resulting in a reduction in the loss. 


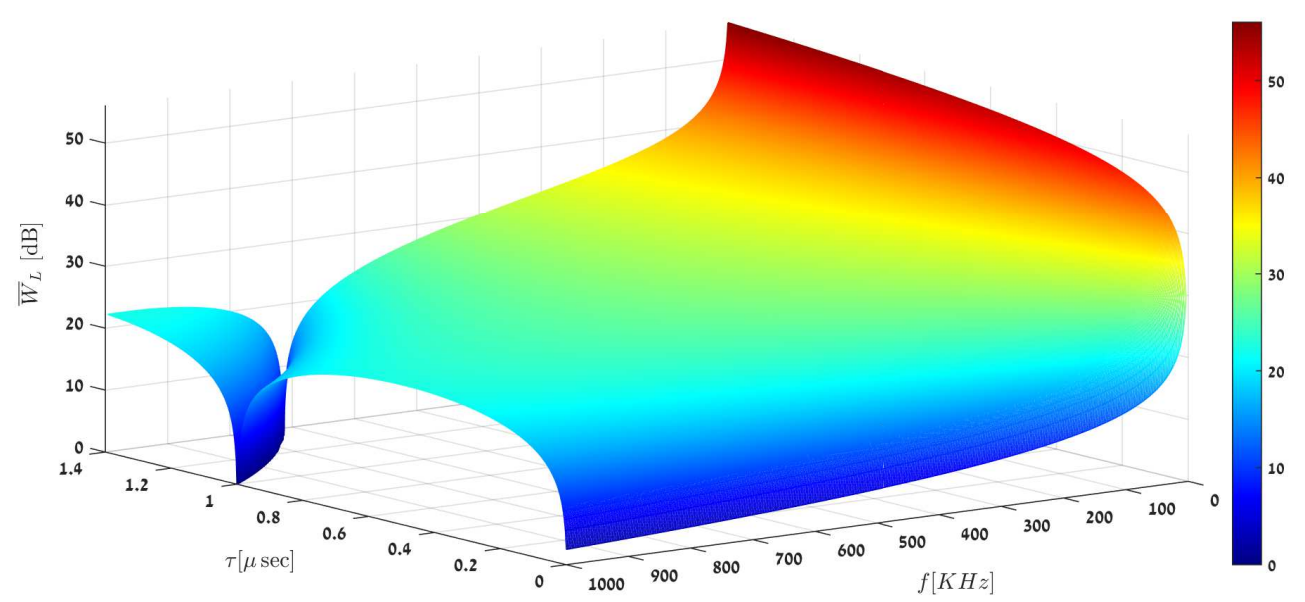

(a)

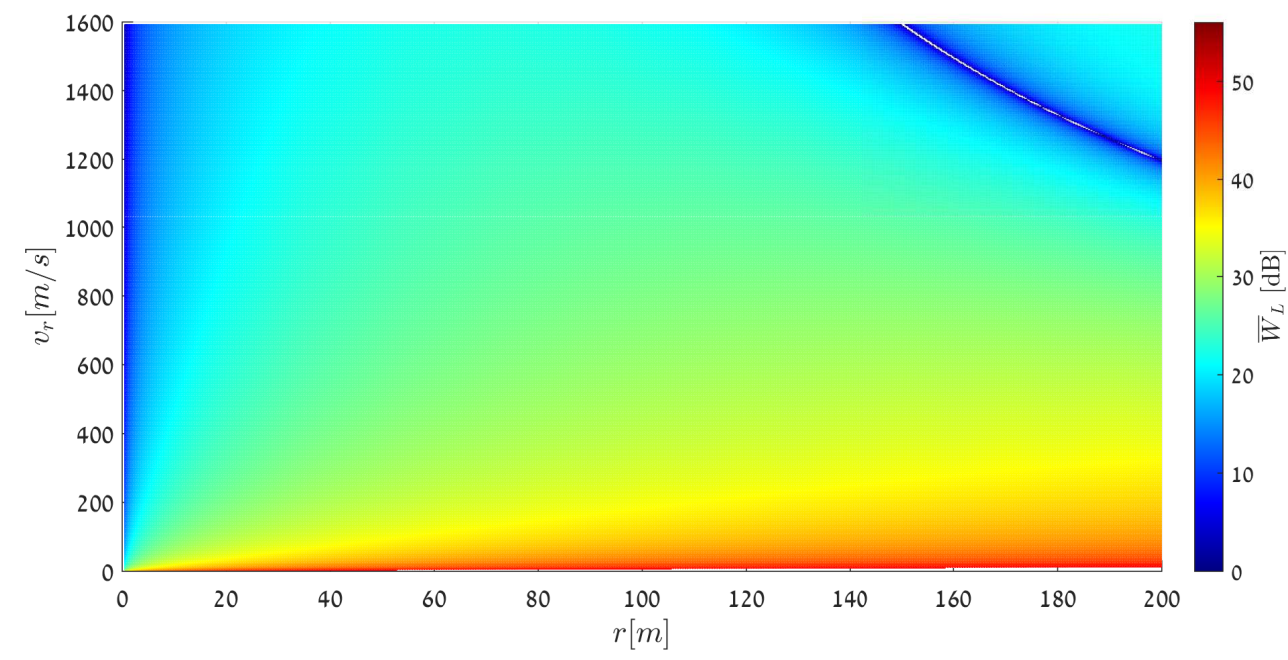

(b)

Figure 5. (a) The resulted three-dimensional plot of the nonstationary coherent integration loss (16) in the time-frequency $(\tau, f)$ domain under quiescent conditions: $\sigma_{1}=10^{-4}$ and $\sigma_{2}=10^{-6}$. (b) The contour plot of the proposed coherent integration loss presented in (a).

An opposite tendency occurs with range, as the target moves away from the radar, the time delay increases, causing a degradation in the coherence between the transmitted and received signals.

A simplified description of the loss as a function of range can be seen in Figure 6 for several target velocities: $v_{r}=100-1100 \mathrm{~m} / \mathrm{s}$.

Table 1 summarizes the coherent integration loss as a function of several ranges and target velocities.

A closer inspection of Figure 6 reveals that the coherent integration obtained for the Agilent oscillator is approximately $35 \mathrm{~dB}$ for a moving target with a radial velocity of $v_{r}=100 \mathrm{~m} / \mathrm{s}$ and is located at a distance of $r_{0}=50 \mathrm{~m}$, whereas a moving target with a supersonic speed of $v_{r}=1100 \mathrm{~m} / \mathrm{s}$ at the same range presents a lower loss of $24 \mathrm{~dB}$.

An interesting phenomenon can be seen for fast-moving targets approaching supersonic velocities. The coherent integration loss shows peaks at intermediate ranges. As the target moves further, the coherent integration loss improves. The nonstationary nature of the phase noise leads to smaller losses in the long range detection of targets. 


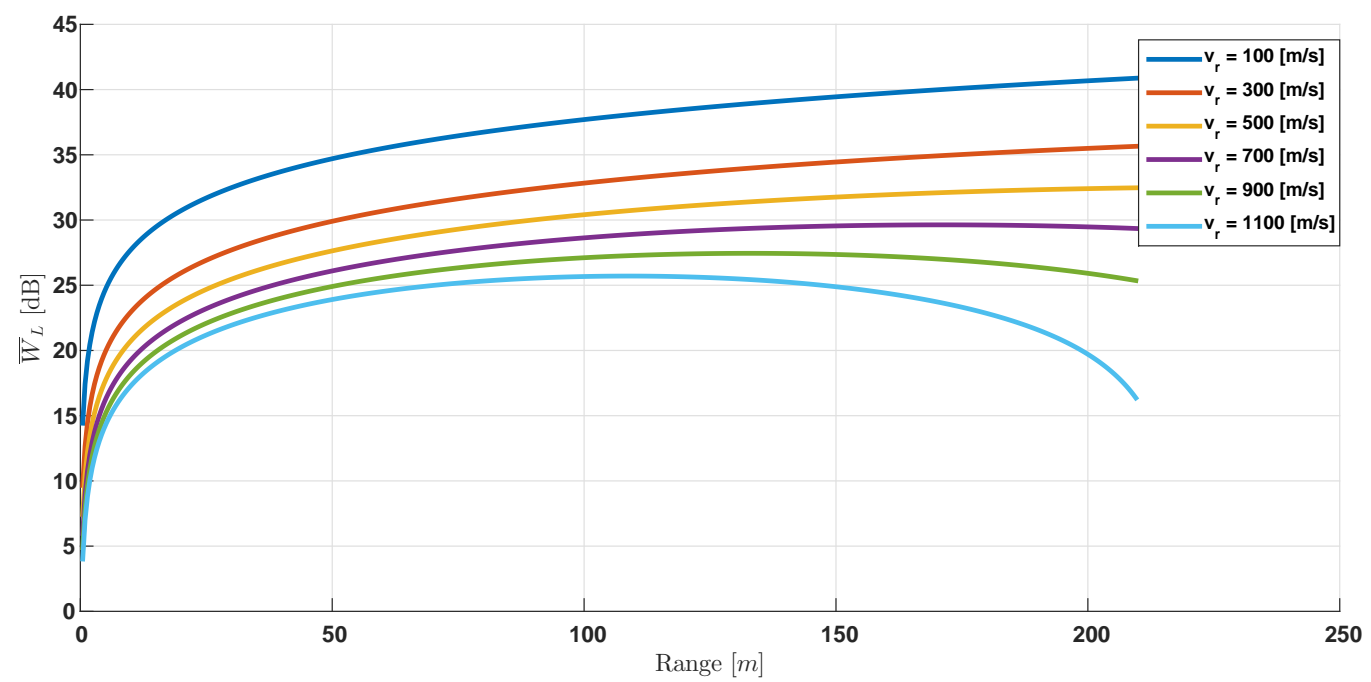

Figure 6. A simplified description of the loss as a function of range for several target velocities between $v_{r}=100$ and $v_{r}=1100 \mathrm{~m} / \mathrm{s}$.

Table 1. A summary of the coherent integration loss as a function of several ranges and target velocities.

\begin{tabular}{lclll}
\hline & \multicolumn{4}{c}{ Range-r $(\mathbf{m})$} \\
\hline & & $\mathbf{2 5}$ & $\mathbf{1 0 0}$ & $\mathbf{2 0 0}$ \\
\hline \multirow{3}{*}{ Radial velocity (m/s) } & $\mathbf{1 0 0}$ & $32 \mathrm{~dB}$ & $37 \mathrm{~dB}$ & $41 \mathrm{~dB}$ \\
& $\mathbf{5 0 0}$ & $24 \mathrm{~dB}$ & $31 \mathrm{~dB}$ & $30 \mathrm{~dB}$ \\
& $\mathbf{1 0 0 0}$ & $21 \mathrm{~dB}$ & $27 \mathrm{~dB}$ & $23 \mathrm{~dB}$ \\
\hline
\end{tabular}

It is interesting to notice that the reduction of the loss peaks for high velocities apply generally for most signal sources and there are no major differences in the source architecture such as cavity oscillators, dielectric resonator oscillators (DROs), PLL, and fractional-N-PLL. The reason for this behavior is the common fundamental nature of the phase noise in oscillators, which declines as the frequency (from the carrier) increases [12]. In the PLL-based frequency synthesizers, the phase noise performance is determined by a superposition of two main noise sources: the reference oscillator noise and the VCO noise. The superposition process saves the fundamental nature described above [19], therefore, the reduction of the loss peaks for high velocities also apply for PLL frequency source architecture. Although, the magnitude and low-power characteristics of the phase noise for different source architectures change from one to another, causing different reduction of the loss peaks for high velocities.

Figure 7a presents a comparison between the proposed approach (16) and the conventional stationary coherent integration loss method given in Equation (18) in [4]: $L=-4.34 \sigma^{2}$, where $L$ denotes the loss in $\mathrm{dB}$ and $\sigma$ represents the stationary phase noise standard deviation. The green plane is the constant stationary coherent integration loss for $\sigma=2 * 10^{-2}$ while the colored three-dimensional plot shows the nonstationary coherent integration loss (16) in the time-frequency $(\tau, f)$ domain under the same conditions described in Figure 5a. The standard deviation $\sigma$ value is chosen so that the same total amount of noise power (as was taken in Figure 5a) will also be available for the stationary model simulation. Figure $7 \mathrm{~b}$ illustrates the same comparison presented above (Figure 7a) in a different angle in order to demonstrate the difference from another point of view. It can be seen that whereas the stationary phase noise method yields a constant value for the coherent integration loss for a given frequency source, the proposed nonstationary coherent integration loss approach yields a varying loss value (16). The nonstationary expression enables the high-resolution radar designer to estimate the loss caused by the local oscillator phase noise for a large range of target velocities. Moreover, the proposed expression (16) can also 
help the radar designer to estimate the loss variation for a specific range-velocity curve in real-time that fits the target trajectory. The resulted real-time loss estimation made by the "Wigner integration" block can be used as an input to a threshold adjustment process in the radar final "Decision" block, as described in Figure 1.

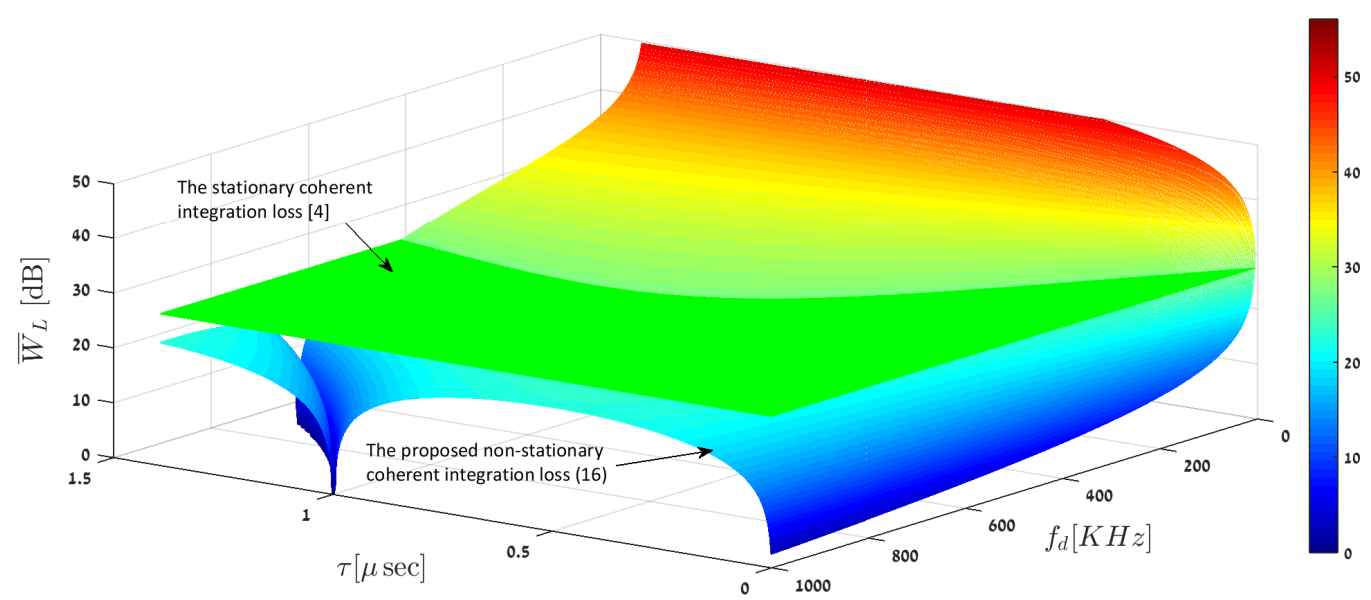

(a)

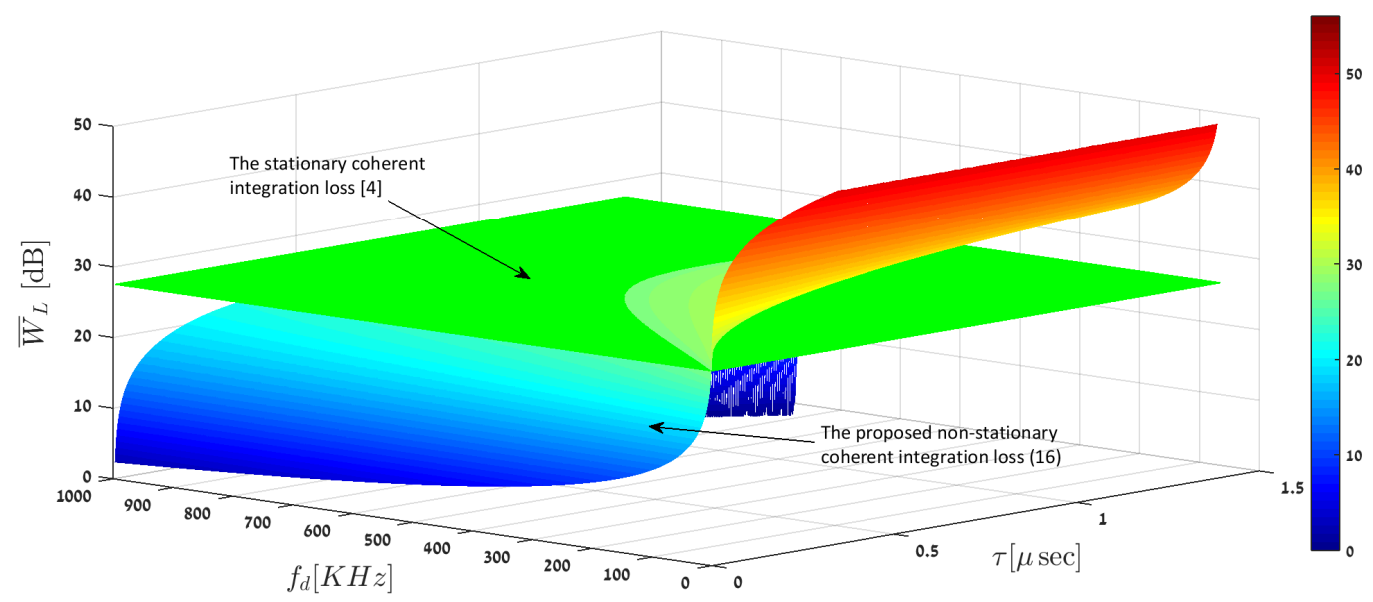

(b)

Figure 7. (a) A comparison between the resulted nonstationary coherent integration loss (16) in the time-frequency $(\tau, f)$ domain vs. the stationary coherent integration loss proposed by [4]. (b) An illustration of the same comparison presented in Figure 7a from a different point of view.

In the following, we will demonstrate our theory with comparison to the stationary coherent integration loss approach, as presented in [4]. Balal et al. [16] presented two experiments with measurement results obtained with the setup illustrated in Figure 3 . The demonstration will be performed by numerical simulation according to those experimental test results. Hereafter, the standard deviation value will be as above $\left(\sigma=2 * 10^{-2}\right)$ in order to maintain a fair comparison between considering and neglecting nonstationary phase noise. In the first experiment, an optical gate system measured the instantaneous velocity of a bullet fired from a gun at an initial speed of about $1200 \mathrm{~m} / \mathrm{s}$. The velocity of the bullet fades as it approaches the radar. Figure 8a shows the velocity $v_{r}$ as a function of $r$. 


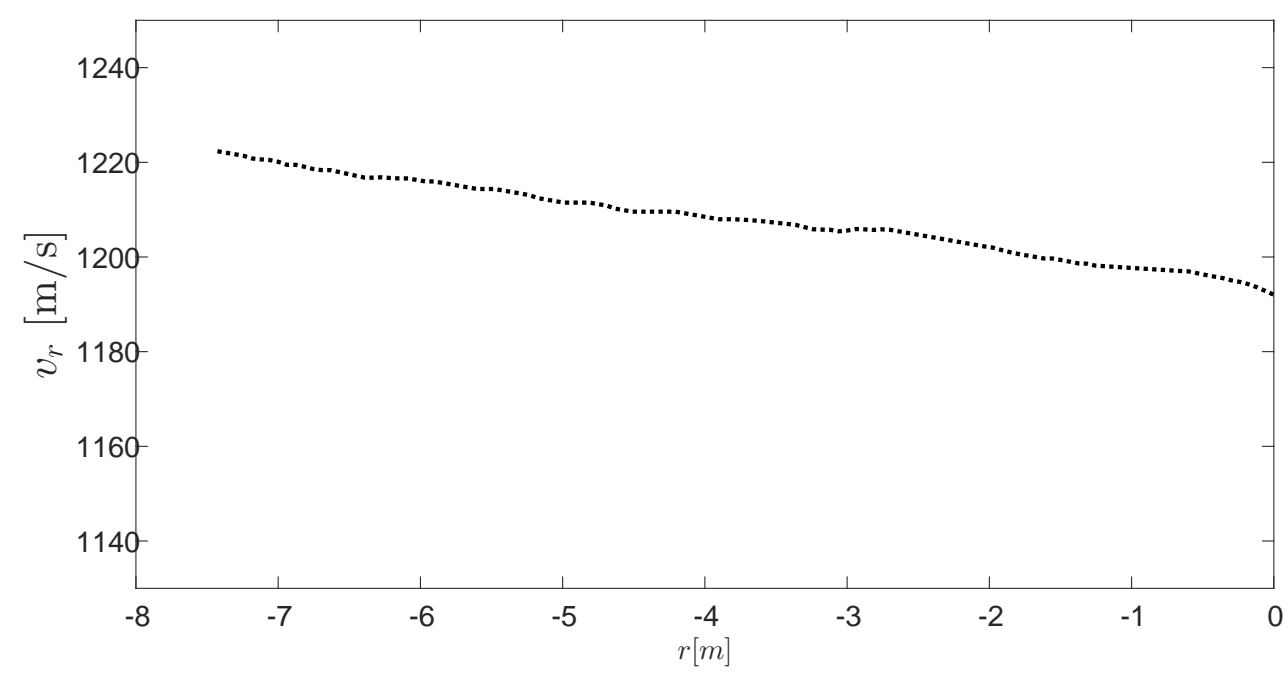

(a)

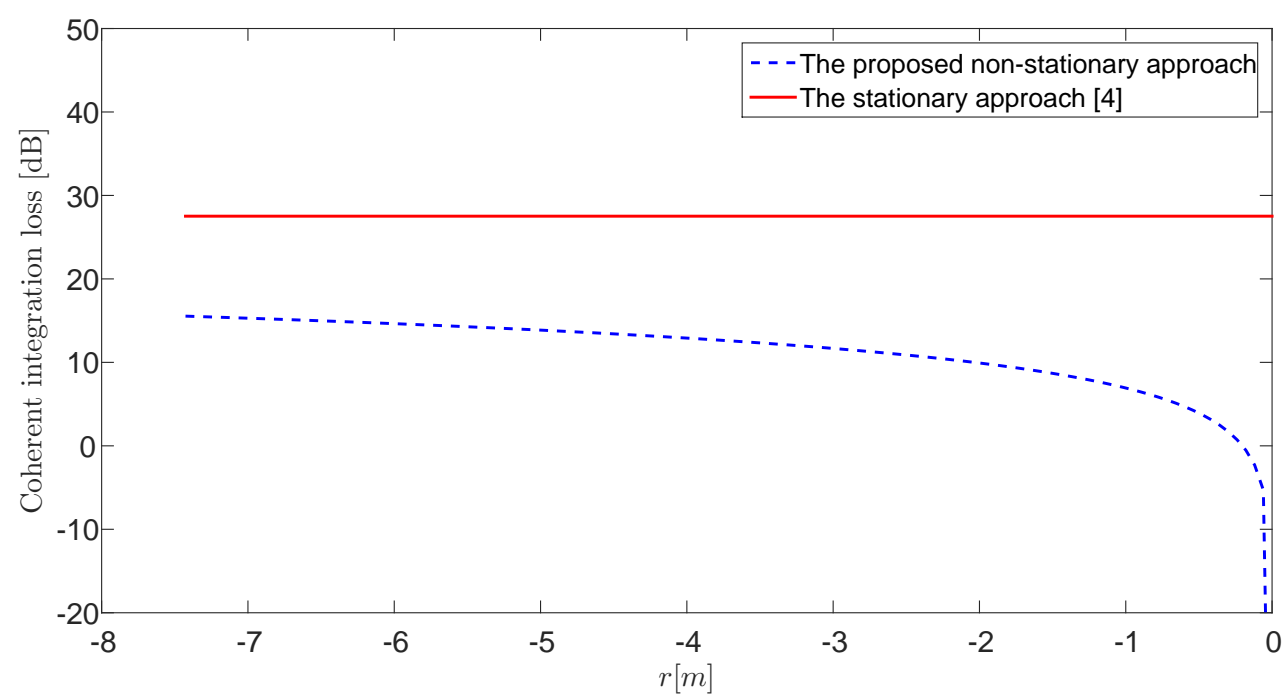

(b)

Figure 8. The first experiment: (a) The instantaneous velocity of a small target dependent on $r$. (b) A comparison between the resulted nonstationary coherent integration loss (16) vs. the stationary coherent integration loss proposed by [4]. Both numerical simulations were performed based on the first experiment test results.

The point at $0 \mathrm{~m}$ on the $x$-axis represents the location of the radar. Figure $8 \mathrm{~b}$ presents the numerical simulation of the proposed nonstationary coherent integration loss (16) vs. the conventional stationary coherent integration loss [4]. Both curves are presented as a function of $r$. In the second experiment, a bullet of a different shape was fired from the gun at a speed of about $500 \mathrm{~m}$.

Figure 9a shows the velocity $v_{r}$ as a function of $r$, while in Figure $9 \mathrm{~b}$, we present the numerical simulation of the proposed nonstationary coherent integration loss (16) vs. the constant stationary coherent integration loss [4]. Both curves are presented as functions of $r$.

These results show that while the conventional stationary coherent integration loss failed to follow the nonstationary process, the proposed theory demonstrated real-time, high-accuracy analysis of short-term phase instabilities. Therefore, in order to increase the radar probability of detection, the proposed approach can be used as an input to a real-time threshold adjustment process in the radar's final decision block. 


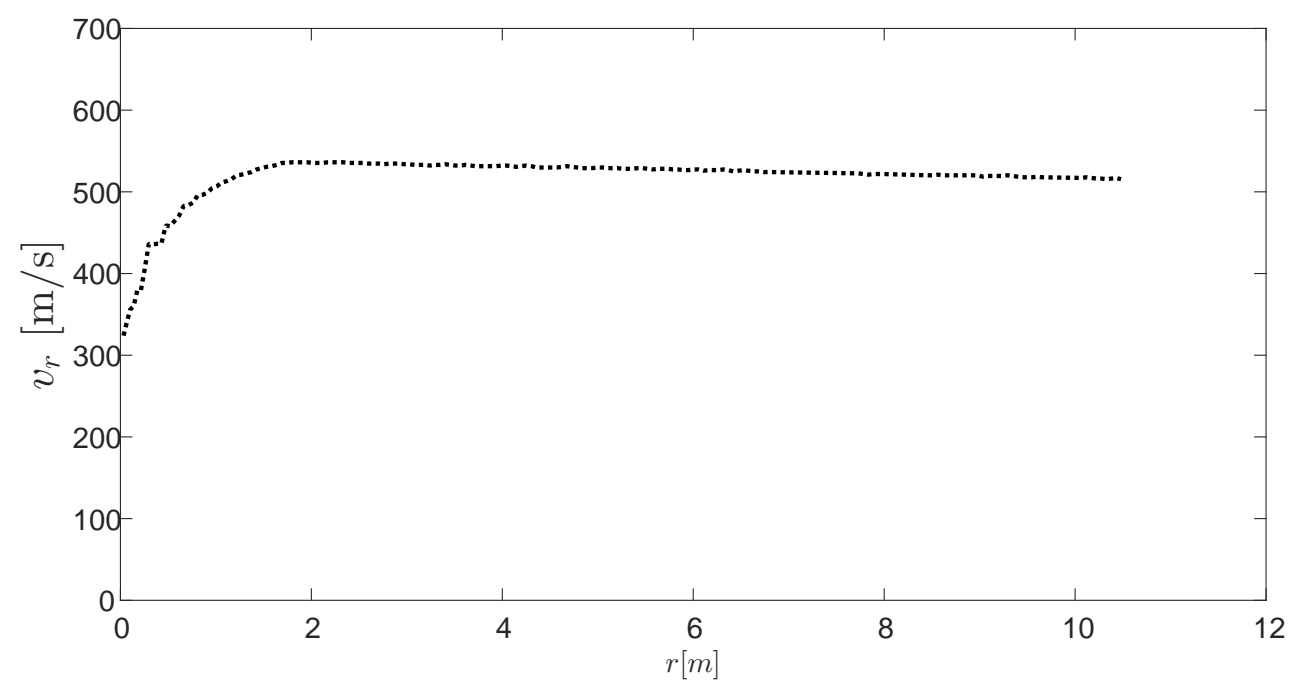

(a)

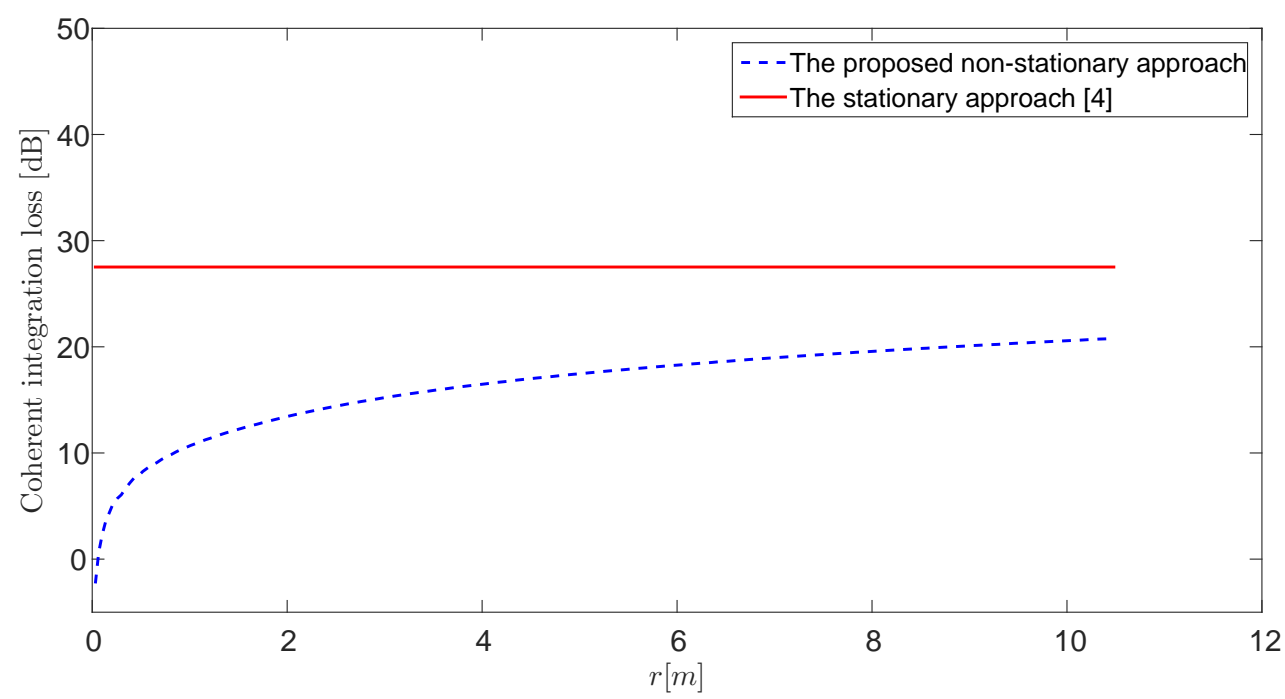

(b)

Figure 9. The second experiment: (a) The instantaneous velocity of another bullet dependent on $r$. (b) A comparison between the resulted nonstationary coherent integration loss (16) vs. the stationary coherent integration loss proposed by [4]. Both numerical simulations were performed based on the second experiment test results.

\section{Summary and Conclusions}

The coherent integration loss characterization of high-resolution radars due to the nonstationary phase noise generated by the local oscillator has been presented herein. Wigner-Ville distribution was employed to express the coherent integration loss in the time-frequency domain. We derived an analytic expression for the coherent integration loss for nonstationary phase noise. While the common characterization of integration loss failed to follow nonstationary processes, the Wigner-Ville distribution demonstrated highaccuracy analysis of short-time phase instabilities and a comprehensive description of their evolution for high-resolution broadband radars. The proposed approach was applied for evaluating the dynamics of coherent integration loss in a high-resolution radar operating in millimeter wavelengths. It is demonstrated that time-frequency analysis exposes the effect of instantaneous, nonstationary phase variations and frequency deviations generally seen in oscillators. Nonstationary phase noise is shown to affect radar performance in scenarios where high-resolution capabilities are required for target detection and tracking. It is interesting to note that the time-frequency analysis presented here indicates that 
variations in the coherent integration loss appears in high-velocity targets moving at subor supersonic velocities.

The new technique allows usage of loss information in regulating the decision threshold. It can help radar systems to evaluate the nonstationary integration loss in real time and modify the decision threshold correspondingly, in order to maximize the radar's detection reliability.

Author Contributions: Conceptualization, C.L., M.P. and Y.P.; methodology, C.L.; software, C.L.; validation, C.L., M.P. and Y.P.; formal analysis, C.L.; investigation, C.L.; resources, C.L., M.P. and Y.P.; data curation, C.L.; writing—original draft preparation, C.L., Y.P. and M.P.; writing-review and editing, C.L., Y.P. and M.P.; visualization, Y.P. and M.P.; supervision, M.P. and Y.P.; project administration, C.L. All authors have read and agreed to the published version of the manuscript.

Funding: This research received no external funding.

Institutional Review Board Statement: Not applicable.

Informed Consent Statement: Not applicable.

Data Availability Statement: The data used to support the findings of this study are included within the article.

Conflicts of Interest: The authors declare no conflict of interest.

\section{Appendix A}

Provided in this appendix are details of the expression for $\bar{W}_{L}(\tau, f)$.

Substituting (6) into (15) yields

$$
\varphi(t) \varphi(t-\tau)=\omega_{0}^{2}\left[y_{0} t+\sigma_{1} B_{H_{1}}(t)+\sigma_{2} \int_{0}^{t} B_{H_{2}}(s) d s\right]\left[y_{0}(t-\tau)+\sigma_{1} B_{H_{1}}(t-\tau)+\sigma_{2} \int_{0}^{t-\tau} B_{H_{2}}(s) d s\right]
$$

$\left\{B_{H_{i}}(t), t \geq 0\right\}, i=1,2$ are two independent, standard Wiener processes, so we can introduce (A1) as

$$
\varphi(t) \varphi(t-\tau)=\omega_{0}^{2}\left[y_{0}^{2} t^{2}-y_{0}^{2} t \tau+\sigma_{1}^{2} B_{H_{1}}(t) B_{H_{1}}(t-\tau)+\sigma_{2}^{2} \int_{0}^{t} B_{H_{2}}(s) d s \int_{0}^{t-\tau} B_{H_{2}}(s) d s\right] .
$$

Therefore, the expectation value of (A2) is given by

$$
E\{\varphi(t) \varphi(t-\tau)\}=\omega_{0}^{2}\left[y_{0}^{2} t^{2}-y_{0}^{2} t \tau+\sigma_{1}^{2} E\left\{B_{H_{1}}(t) B_{H_{1}}(t-\tau)\right\}+\sigma_{2}^{2} E\left\{\int_{0}^{t} B_{H_{2}}(s) d s \int_{0}^{t-\tau} B_{H_{2}}(s) d s\right\}\right] .
$$

The standard Wiener process $\left\{B_{H}(t), t \geq 0\right\}$ has the following covariance function $[20,21]$ :

$$
E\left\{B_{H}(t) B_{H}(s)\right\}=\frac{1}{2}\left(|t|^{2 H}+|s|^{2 H}-|t-s|^{2 H}\right) .
$$

In our case, the process $B_{H}(t)$ is the standard Brownian motion, $(H=0.5)$; therefore, by substituting (A4) into the middle term of (A3), we get

$$
E\{\varphi(t) \varphi(t-\tau)\}=\omega_{0}^{2}\left\{y_{0}^{2} t^{2}-y_{0}^{2} t \tau+\frac{\sigma_{1}^{2}}{2}(|t|+|t-\tau|-|\tau|)+E\left\{\sigma_{2} \int_{0}^{t} B_{H_{2}}(s) d s \sigma_{2} \int_{0}^{t-\tau} B_{H_{2}}(s) d s\right\}\right\}
$$

Now, the expectation value is inserted into the joint integral argument on the last term of (A3):

$$
E\{\varphi(t) \varphi(t-\tau)\}=\omega_{0}^{2}\left\{y_{0}^{2} t^{2}-y_{0}^{2} t \tau+\frac{\sigma_{1}^{2}}{2}(|t|+|t-\tau|-|\tau|)+\sigma_{2}^{2} \int_{0}^{t} \int_{0}^{t-\tau} E\left\{B_{H_{2}}(s) B_{H_{2}}\left(s^{\prime}\right)\right\} d s d s^{\prime}\right\} .
$$


Next, the expectation value of the last term of (A6) is calculated, again, by using (A4):

$$
E\{\varphi(t) \varphi(t-\tau)\}=\omega_{0}^{2}\left\{y_{0}^{2} t^{2}-y_{0}^{2} t \tau+\frac{\sigma_{1}^{2}}{2}(|t|+|t-\tau|-|\tau|)+\sigma_{2}^{2} \int_{0}^{t} \int_{0}^{t-\tau} \frac{1}{2}\left(|s|+\left|s^{\prime}\right|-\left|s-s^{\prime}\right|\right) d s d s^{\prime}\right\} .
$$

After some trivial integral calculation, (A7) can be written in its final form:

$$
\begin{aligned}
& E\{\varphi(t) \varphi(t-\tau)\}= \\
& \omega_{0}^{2}\left\{y_{0}^{2} t^{2}-y_{0}^{2} t \tau+\frac{\sigma_{1}^{2}}{2}(|t|+|t-\tau|-|\tau|)\right. \\
& \left.+\frac{\sigma_{2}^{2}}{4}|t-\tau|(t-\tau) t+\frac{\sigma_{2}^{2}}{4}|t| t(t-\tau)+\frac{\sigma_{2}^{2}}{12}\left[|\tau| \tau^{2}-|-t+\tau|(-t+\tau)^{2}-|t| t^{2}\right]\right\} .
\end{aligned}
$$

Next, the closed form expression for the main term of (15) ( $\left.\int_{-\infty}^{\infty} E\{\varphi(t) \varphi(t-\tau)\} e^{-i 2 \pi f t} d t\right)$ is presented by solving the Fourier transformation of (A8).

The seven terms of (A8) are defined as follows:

$$
\begin{aligned}
& a_{12}(t, \tau)=y_{0}^{2} t^{2}-y_{0}^{2} t \tau \\
& b_{12}(t, \tau)=\frac{\sigma_{1}^{2}}{2}(|t|+|t-\tau|-|\tau|) \\
& c_{12}(t, \tau)=\frac{\sigma_{2}^{2}}{4}|t-\tau|(t-\tau) t \\
& d_{12}(t, \tau)=\frac{\sigma_{2}^{2}}{4}|t| t(t-\tau) \\
& e_{12}(t, \tau)=\frac{\sigma_{2}^{2}}{12}|\tau| \tau^{2} \\
& f_{12}(t, \tau)=-\frac{\sigma_{2}^{2}}{12}|-t+\tau|(-t+\tau)^{2} \\
& g_{12}(t, \tau)=-\frac{\sigma_{2}^{2}}{12}|t| t^{2} .
\end{aligned}
$$

Therefore, we can state that (A8) can be written as

$$
\begin{gathered}
E\{\varphi(t) \varphi(t-\tau)\}=\omega_{0}^{2}\left[a_{12}(t, \tau)+b_{12}(t, \tau)+c_{12}(t, \tau)+d_{12}(t, \tau)+e_{12}(t, \tau)+f_{12}(t, \tau)+g_{12}(t, \tau)\right] . \\
\text { Next, the closed form expression for } \int_{-\infty}^{\infty} E\{\varphi(t) \varphi(t-\tau)\} e^{-i 2 \pi f t} d t \text { is presented by } \\
\text { solving the Fourier transformation of (A9): } \\
\int_{-\infty}^{\infty} E\{\varphi(t) \varphi(t-\tau)\} e^{-i 2 \pi f t} d t= \\
\omega_{0}^{2} \int_{-\infty}^{\infty}\left[a_{12}(t, \tau)+b_{12}(t, \tau)+c_{12}(t, \tau)+d_{12}(t, \tau)+e_{12}(t, \tau)+f_{12}(t, \tau)+g_{12}(t, \tau)\right] e^{-i 2 \pi f t} d t \\
=\left(2 \pi v_{0}\right)^{2}\left[A_{12}(t, f)+B_{12}(t, f)+C_{12}(t, f)+D_{12}(t, f)+E_{12}(t, f)+F_{12}(t, f)+G_{12}(t, f)\right],
\end{gathered}
$$

where $A_{12}(t, f), B_{12}(t, f), C_{12}(t, f), D_{12}(t, f), E_{12}(t, f), F_{12}, G_{12}(t, f)$ denote the Fourier transformation of $a_{12}, b_{12}, c_{12}, d_{12}, e_{12}, f_{12}, g_{12}(t, \tau)$ given in (A9), respectively.

Finally, by solving the Fourier transformation of the seven terms stated in (A9) and substituting their closed form expressions into (15), we get (16).

\section{References}

1. Bassem, R.M. Radar Systems Analysis and Design Using MATLAB deciBEL Research; Chapman and Hall/CRC Inc.: Boca Raton, FL, USA, 2013; ISBN 978-1-4398-8495-9.

2. Yu, J.; Xu, J.; Peng, Y. Upper Bound of Coherent Integration Loss for Symmetrically Distributed Phase Noise. Signal Process. Lett. 2008, 15, 661-664.

3. Doerry, A.W. Radar Receiver Oscillator Phase Noise; Sandia Report SAND2018-3614; Sandia National Laboratories: Albuquerque, NM, USA; Livermore, CA, USA, 2018.

4. Richards, M.A. Coherent Integration Loss Due to White Gaussian Phase Noise. IEEE Signal Process. Lett. 2003, 10, 7. [CrossRef]

5. Nebel, M.; Lankl, B. Oscillator phase noise as a limiting factor in stand-alone GPS-indoor navigation. In Proceedings of the 2015 IEEE International Conference on Information and Automation, Lijiang, China, 8-10 August 2010; pp. 1-8.

6. Galleani, L.; Member, S.; Tavella, P.; Member, S. Detection of Atomic Clock Frequency Jumps With the Kalman Filter. IEEE Trans. Ultrason. Ferroelectr. Freq. Control 2012, 59, 3. [CrossRef] [PubMed] 
7. Galleani, L.; Tavella, P. The characterization of clock behavior with the dynamic Allan variance. In Proceedings of the IEEE International Frequency Control Symposium and PDA Exhibition Jointly with the 17th European Frequency and Time Forum, Tampa, FL, USA, 4-8 May 2003; pp. 5-8.

8. Sesial, I.; Galleani, T.P. Application of the Dynamic Allan Variance for the Characterization of Space Clock Behavior. IEEE Trans. Aerosp. Electron. Syst. 2011, 47, 2.

9. Keysight, T. Analyzing Frequency Stability in the Frequency and Time Domains; Application Note; Keysight Technologies: Santa Rosa, CA, USA, 2014.

10. Zucca, C.; Tavella, P. The clock model and its relationship with the Allan and related variances. IEEE Trans. Ultrason. Ferroelectr. Freq. Control 2005, 52, 289-296. [CrossRef] [PubMed]

11. Zucca, C. A mathematical model for the atomic clock error in case of jumps. arXiv 2015, arXiv:1506.01080v1. [CrossRef]

12. Rubiola, E. Phase Noise and Frequency Stability in Oscillators; Cambridge University Press: Cambridge, UK, 2009.

13. Cohen, L. Time-Frequency Analysis; Prentice Hall: Upper Saddle River, NJ, USA, 1994.

14. Ville, J. Theorie et applications de la notion de signal analytique. Cables Transm. 1948, 2, 61-74.

15. Wigner, E.P. On the quantum correction for thermodynamic equilibrium. Phys. Rev. 1932, 40, 749-759. [CrossRef]

16. Balal, N.; Balal, Y.; Richter, Y.; Pinhasi, Y. Detection of Low RCS Supersonic Flying Targets with a High-Resolution MMW Radar. Sensors 2020, 20, 3284. [CrossRef] [PubMed]

17. KEYSIGHT, 83623B High Power Swept-Signal Generator. Available online: https://www.keysight.com/en/pd-1000001863 (accessed on 20 February 2021).

18. Analog Devices, ADF4371-Microwave Wideband Synthesizer with Integrated VCO. Available online: https://www.analog. com/en/products/adf4371.html\#product-overview (accessed on 25 March 2021).

19. Valenta, V.; Baudoin, G.; Villegas, M. Phase Noise Analysis of PLL Based Frequency Synthesizers for Multi-Radio Mobile Terminals. In Proceedings of the Third International Conference on Cognitive Radio Oriented Wireless Networks and Communications, CrownCom 2008, Singapore, 15-17 May 2008; ISBN 978-1-4244-2302-6.

20. Fima, C.K. Introduction to Stochastic Calculus with Applications; Imperial College Press: London, UK, 1998.

21. Steven, E.S. Stochastic Calculus for Finance II: Continuous-Time Models; Springer: Berlin/Heidelberg, Germany, 2004. 